\title{
Salivary Circular RNAs Hsa_Circ_0001874 and Hsa_Circ_0001971 as Novel Biomarkers for the Diagnosis of Oral Squamous Cell Carcinoma
}

\author{
Si-Yu Zhao $\quad$ Jun Wang ${ }^{b}$ Shao-Bo Ouyanga $\quad$ Zi-Kun Huang ${ }^{c}$ Lan Liao ${ }^{a}$ \\ aDepartment of Oral Prosthodontics, Affiliated Stomatological Hospital of Nanchang University, Jiangxi \\ Provinial Key Laboratory of Oral Biomedicine, Nanchang, 'b Oral and Maxillofacial Surgery, Second \\ Affiliated Hospital of Nanchang University, Nanchang, 'Department of Clinical Laboratory, the First \\ Affiliated Hospital of Nanchang University, Nanchang, China
}

\section{Key Words}

Oral squamous cell carcinoma $•$ Circular RNAs $•$ Salivary $•$ Biomarker • Diagnosis

\begin{abstract}
Background/Aims: Recent studies have demonstrated that circular RNAs (circRNAs) can serve as potential molecular markers for disease diagnosis. However, little is known about their diagnostic potential for oral squamous cell carcinoma (OSCC). This study aimed to determine the expression of circRNAs in the saliva of OSCC patients to identify novel biomarkers for OSCC screening. Methods: Microarray screening of circRNA was performed to identify differentially expressed circRNAs in saliva from 3 OSCC patients compared with 3 healthy controls. Quantitative reverse transcription polymerase chain reaction ( $q R T-P C R$ ) was used to validate the results, and the association between these confirmed salivary circRNAs and clinicopathological features was analyzed using the chi-squared test. A receiver operating characteristic (ROC) curve was constructed to evaluate the diagnostic value of the circRNAs identified. Preoperative expression and postoperative expression (1 month after the surgery) of hsa_circ_0001874 and hsa_circ_0001971 was also determined. Results: Our results indicated 12 upregulated and 20 downregulated circRNAs in the saliva from the OSCC patients compared with that from the healthy controls. Among the differentially expressed circRNAs, hsa_circ_0001874, hsa_circ_0001971, and hsa_circ_0008068 were upregulated and hsa_circ_0000140, hsa_circ_0002632, and hsa_circ_0008792 were downregulated in the OSCC group versus the healthy group. Clinical data indicated that salivary hsa_circ_0001874 was correlated with TNM stage $(P=0.006)$ and tumor grade $(P=0.023)$ and that hsa_circ_0001971 was correlated with TNM stage $(P=0.019)$. The combination of hsa_circ_0001874 and hsa_ circ_0001971 showed an area under the ROC curve of 0.922 (95\% confidence interval, $0.883-0.961 ; P<0.001)$. The risk score based on the combination of hsa_circ_0001874 and $\mathrm{S}$. Zhao and J. Wang contributed equally to this work.

\begin{tabular}{ll}
\hline Lan Liao & Dept. of Oral Prosthodontics, Aff. Stomatological Hosp. of Nanchang Univ. \\
& Jiangxi Provinial Key Lab. of Oral Biomedicine, 49 Fu zhou lu, Nanchang 330006 (China) \\
& Tel. (+86) 0791-86363685, Fax (+86) 0791-86361141, E-Mail liaolan5106@163.com
\end{tabular}
\end{abstract}


Zhao et al.: Salivary CircRNAs and OSCC

hsa_circ_0001971 also discriminated patients with OSCC from patients with oral leukoplakia $(P<0.001)$. Moreover, the expression levels of salivary hsa_circ_0001874 and hsa_circ_0001971 were clearly decreased in the postoperative samples compared with preoperative samples $(P<0.001)$. Conclusions: This is the first study to demonstrate the potential of salivary hsa circ_0001874 and hsa_circ_0001971 as biomarkers for the diagnosis of OSCC.

(C) 2018 The Author(s)

Introduction

Published by S. Karger AG, Basel

Oral squamous cell carcinoma (OSCC) is one of the most common malignant tumors in the oral and maxillofacial region. It is estimated to be the ninth most common cancer worldwide, and its incidence rate has been showing an increasing trend [1]. Despite advances in medical and surgical treatments, most OSCC patients are diagnosed at an advanced stage and have a poor prognosis, with an overall 5-year survival rate of 50-60\% [2]. Classic tumor markers such as serum squamous cell carcinoma antigen, carbohydrate antigen 19-9, and carcinoembryonic antigen are used in the management of patients with OSCC. However, due to insufficient diagnostic sensitivity and specificity, they have limited utility in the early detection of this disease [3]. Therefore, new biomarkers with high sensitivity and specificity should be explored for the early detection of OSCC.

Circular RNAs (circRNAs) are a class of endogenous RNAs that are characterized by covalently closed loop structures without a $5^{\prime}$ cap or a $3^{\prime}$ poly(A) tail [4]. There is increasing evidence that circRNAs play important roles in the regulation of gene expression at the posttranscriptional level [5-7]. Accordingly, it has been demonstrated that many circRNAs serve as competing endogenous RNAs to bind with microRNAs (miRNAs) and inhibit the activity and function of the targeted miRNAs [8, 9]. With the increasing number of studies on circRNAs, circRNAs have been shown to be involved in the development of several types of diseases, including cancer [10], cardiovascular disease [11], and neurological disorders [12]. Unlike linear RNA, circRNAs have a special circular covalently bonded structure, which facilitates their higher stability and resistance to RNA exonucleases [13]. The expression of circRNAs in human blood and gastric fluid also increases their potential as disease biomarkers [14]. For example, Zhao et al [15]. discovered that peripheral blood hsa_circ_0124644 can be used as a diagnostic biomarker of coronary artery disease and Yin et al [16]. reported that plasma hsa_circ_0001785 can serve as a diagnostic biomarker for breast cancer detection. Saliva contains an array of analytes (protein, mRNA, and DNA) that can be used as biomarkers for translation and clinical applications [17]. Using bioinformatics analysis, Bahn et al [18]. identified many circRNAs in human saliva and suggested salivary circRNAs are potential non-invasive biomarkers for the diagnosis of human diseases.

In this study, we used microarray analysis to compare the expression profile of circRNAs in the saliva of OSCC patients and matched control subjects and then confirmed our findings in larger independent cohorts. Our results indicate the potential of salivary hsa_circ_0001874 and hsa_circ_0001971 as biomarkers for the diagnosis of OSCC.

\section{Materials and Methods}

\section{Patient variables}

A total of 93 consecutive patients with OSCC were recruited from the Affiliated Stomatological Hospital of Nanchang University between January 2016 and May 2017. All were newly diagnosed and previously untreated (e.g., no surgery, chemotherapy, or radiotherapy). Their clinicopathological data included age, sex, location, size, and grade of tumor, distant metastasis, and TNM stage. In addition, 70 subjects who were clinically and pathologically diagnosed with oral leukoplakia (OLK) were recruited for this study. To serve as controls, 85 individuals who sought a routine health check-up in the same period and did not have any oral diseases or other cancerous diseases were recruited. Among the participants, 3 OSCC patients and 3 age- and sex-matched healthy controls were recruited for microarray analysis. Other samples were used 
Zhao et al.: Salivary CircRNAs and OSCC

to verify the microarray results by quantitative reverse transcription polymerase chain reaction (qRT-PCR).

This study was approved by the Ethics Committee of the Affiliated Stomatological Hospital of Nanchang University and was conducted in accordance with the tenets of the Helsinki Declaration. Informed consent was obtained from all participants prior to participation.
Table 1. Primers used for qRT-PCR analysis of circRNA and mRNA levels

\begin{tabular}{|c|c|c|}
\hline Name & Primer sequence $5^{\prime}-3^{\prime}$ & Product size (bp) \\
\hline \multirow{2}{*}{$\beta$-actin } & F: CATGTACGTTGCTATCCAGGG & 250 \\
\hline & R: CTCCTTAATGTCACGCACGAT & \\
\hline \multirow[t]{2}{*}{ hsa_circ_0001874 } & F: TTGGCTCTCCTGCTGTGC & 122 \\
\hline & R: GGTCATCCACAATCAGCCCA & \\
\hline \multirow{2}{*}{ hsa_circ_0001971 } & F: GCTGCCTTAACTTACATGCCC & 158 \\
\hline & R: ACTTTGTGGCTCCTGGATAACT & \\
\hline \multirow{2}{*}{ hsa_circ_0008068 } & F: TTCCCGTGGGACATTGATGA & 154 \\
\hline & R: AGTCGTAATTTCCAAGAAGGGCA & \\
\hline hsa_circ_0000140 & $\begin{array}{l}\text { F: CCGGCATTACCTACTGGAGTC } \\
\text { R: CCTTCCACCTTCTCCTTGACA }\end{array}$ & 161 \\
\hline hsa_circ_0002632 & F: ACCATGGATGAAACACGCAA & 125 \\
\hline hsa_circ_0008792 & $\begin{array}{l}\text { R: CTCGACCAGGATCAAAGCGA } \\
\text { F: CCAAGGTGAACAATGCGACG } \\
\text { R: TGAGATGTGTGATCCATCCAAT }\end{array}$ & 151 \\
\hline
\end{tabular}

Collection of saliva samples and extraction of total RNA

Before saliva collection, each individual fasted, was prohibited from drinking and smoking, and had not brushed their teeth, rinsed, or flossed for at least $2 \mathrm{~h}$. Each saliva sample was centrifuged at $4^{\circ} \mathrm{C}$ at $3000 \times \mathrm{g}$ for $15 \mathrm{~min}$, after which the supernatant was transferred to a $1.5-\mathrm{mL}$ EP tube. The supernatant was centrifuged at $4^{\circ} \mathrm{C}$ at $12000 \times \mathrm{g}$ for $10 \mathrm{~min}$ and the supernatant was collected. After separation, saliva samples were stored at $-80^{\circ} \mathrm{C}$ until total RNA extraction. Total RNA was isolated using the miRNeasy Mini Kit (Qiagen, Hilden, Germany) according to the manufacturer's protocol. The RNA concentration was measured using the NanoDrop ${ }^{\mathrm{TM}} 1000$ spectrophotometer (Thermo Fisher Scientific, Waltham, MA, USA).

\section{Microarray analysis of circRNA expression}

The Human CircRNA Array is manufactured by Arraystar Inc. (Rockville, MD, USA). For microarray analysis, total RNA was digested with RNase R (Epicentre Inc., Madison, WI, USA) to remove linear RNAs and enrich circRNAs. The enriched circRNAs were amplified and transcribed into fluorescent circRNAs utilizing a random priming method (Arraystar Super RNA Labeling Kit; Arraystar Inc.). The labeled circRNAs were hybridized to the Arraystar Human circRNA Microarray version (Arraystar Inc.). After washing the slides, the arrays were scanned with the Agilent Scanner G2505C (Agilent Technologies, Santa Clara, CA, USA). Agilent Feature Extraction software (version 11.0.1.1) was used to analyze the acquired array images. Quantile normalization and subsequent data processing were performed using the R software package (R version 3.1.2). The microarray was performed by KangChen Bio-tech (Shanghai, China). Student's $t$-test was performed to determine statistical differences. A false discovery rate (FDR) less than 0.05 was applied to determine the threshold $P$ value. Differentially expressed circRNAs with statistical significance (fold-change $\geq 1.5$ and $P<0.05$ ) between groups were identified using a fold-change cut-off.

\section{Quantitative real-time PCR analysis}

Total RNA (800 ng) from each saliva sample was reverse-transcribed into cDNA with a random primer using the PrimeScript ${ }^{\mathrm{TM}}$ RT Reagent Kit (Takara Bio Inc., Tokyo, Japan) according to the manufacturer's instructions. To confirm the specificity of PCR amplification, total RNA was pretreated with DNase I to remove trace genomic DNA, and with RNase R (Epicentre Inc.) to remove all linear RNAs before reverse transcription. Then, quantitative real-time (qRT)-PCR was conducted on the ABI 7500 Real-Time PCR System (Applied Biosystems; Thermo Fisher Scientific) using SYBR ${ }^{\circledR}$ Premix Ex Taq ${ }^{\mathrm{TM}}$ II (Takara) in $20 \mu \mathrm{L}$ reactions containing $1 \times$ SYBR Green PCR Master Mix, $0.4 \mu \mathrm{M}$ of each forward and reverse primer, and $1 \mu \mathrm{L}$ cDNA template. The PCR assay was conducted at an initial denaturation step at $95^{\circ} \mathrm{C}$ for 5 min, followed by 40 cycles at $95^{\circ} \mathrm{C}$ for $15 \mathrm{~s}$ and at $60^{\circ} \mathrm{C}$ for $1 \mathrm{~min}$. The melting curve was analyzed to confirm the specificity of amplification and lack of primer dimers. The primers used in qRT-PCR are shown in Table 1. All qRT-PCR experiments were performed in triplicate. qRT-PCR data were analyzed by the $\Delta$ Ct method and normalized to the internal control ( $\beta$-actin). Larger $\Delta$ Ct values indicated lower expression. The specificity of the qRTPCR product of circRNA was determined by electrophoresis, melting curve analysis, and sequencing. Sanger sequencing of the qRT-PCR products confirmed the back-splice junction sequence of circRNA, which was consistent with that shown in the database (http://www.circbase.org/cgi-bin/simplesearch.cgi). 
Statistical analysis

Statistical analysis and graphic presentation were conducted using SPSS version 17.0 (SPSS Inc., Chicago, IL, USA) and GraphPad Prism version 5.0 (GraphPad Software, San Diego, CA, USA). The statistical significance of microarray data was analyzed in terms of fold change using Student's $t$-test, and FDR was calculated to correct the $P$-value. Numerical data are shown as the mean \pm standard error of the mean (SEM). The Mann-Whitney $U$ test or Student's $t$-test was used for statistical analysis, and the Wilcoxon test was used to compare the paired saliva samples before and 1 month after surgery. Receiver operating characteristic (ROC) curves were constructed, and the area under curve (AUC) was used to assess the sensitivity and specificity of the predictive power of using salivary circRNAs as biomarkers for OSCC. The ROC curve was used to classify the results of discriminant analysis and evaluation. An ROC curve requires a binary variable, but the analysis can be applied to a continuous variable by encoding it into two categorical variables. Here, the decision of a cutoff value is made by varying the value and calculating the sensitivity (sensitivity) and false positive rate (1-Specificity (specificity)), with sensitivity along the vertical axis, to calculate the error as described in Wang et al [19].. Curves are drawn to connect the points, and the area under the curve is calculated, with larger areas indicating greater error. The AUC is calculated as follows. First, the scores are arranged in order of descending score, and the maximum score corresponds to the sample if the rank is $n$, the second-highest score corresponds to a rank of $n-1$, and so on. The ranks of all positive classes are summed, and the average of $M$ and $1+M$ is subtracted, where $M$ is the number of positive class samples. This intermediate score is then divided by the product $M \times N$. This is written as the following.

$$
A U C=\frac{\sum_{i \in \text { positiveclass }} \operatorname{rank}_{i}-\frac{M(1+M)}{2}}{M \times N}
$$

In addition, special attention is required for the case of a tie score. Two samples with the same score must be given the same rank (whether the scores are in the same sample or in different types of samples). The samples thus need equal rank score averaging, which the above formula achieves. The association between the clinical features and salivary circRNA expression was evaluated by the chi-squared test. $P$ values (two-tailed) less than 0.05 were considered statistically significant.

\section{Results}

\section{CircRNA expression profiles in saliva from OSCC patients}

To identify circRNAs that were differentially expressed in OSCC patients, we performed microarray analysis of circRNAs in the saliva from 3 OSCC patients and 3 age- and sexmatched healthy controls using the Arraystar Human circRNA Microarray. Hierarchical clustering (Fig. 1A) and scatter plot (Fig. 1B) visualization showed that the expression levels of circRNAs were variable between OSCC patients and healthy controls. Differentially expressed circRNAs with statistical significance between the two groups were identified using volcano plot filtering (Fig. 1C): 32 circRNAs were differentially expressed (fold-change $\geq 1.5$ and $P<0.05), 12$ of which were upregulated and 20 of which were downregulated in OSCC patients. The top 20 differentially expressed circRNAs are listed in Table 2. Among the upregulated circRNAs, there were 10 exonic, 1 intronic, and 1 sense overlapping. Among the downregulated circRNAs, there were 17 exonic, 2 intronic, and 1 sense overlapping. To

Table 2. Top 20 differently expressed circRNAs in OSCC patients. Abbreviations: FDR: false discover rate; FC: fold change

\begin{tabular}{ccccccc}
\hline circRNA & P value & FDR & FC & Regulation & circRNA_type & GeneSymbol \\
\hline hsa_circ_0001874 & 0.00901 & 0.14053 & 4.41433 & up & intronic & BICD2 \\
hsa_circ_0001971 & 0.00007 & 0.00784 & 2.86576 & up & exonic & FAM126A \\
hsa_circ008068 & 0.00288 & 0.22297 & 2.17171 & up & exonic & KATNAL1 \\
hsa_circ0003759 & 0.00442 & 0.07613 & 2.01390 & up & exonic & LPP \\
hsa_circ_0005623 & 0.04719 & 0.23760 & 1.83861 & up & exonic & ETFA \\
hsa_circ_0000711 & 0.03860 & 0.20721 & 1.80261 & up & exonic & NFATC3 \\
hsa_circ_0000378 & 0.00559 & 0.08913 & 1.72933 & up & exonic & LRP6 \\
hsa_circ0007646 & 0.00262 & 0.08140 & 1.67597 & up & exonic & DCUN1D4 \\
hsa_circ_0000807 & 0.01924 & 0.16284 & 1.62181 & up & sense overlapping & SEPT9 \\
hsa_circ_0002279 & 0.03099 & 0.19464 & 1.57915 & up & exonic & NPLOC4 \\
hsa_circ_0000140 & 0.00242 & 0.08804 & 2.64636 & down & exonic & KIAA0907 \\
hsa_circ_0002632 & 0.00205 & 0.08204 & 2.58023 & down & exonic & STIL \\
hsa_circ0008792 & 0.02669 & 0.19109 & 2.41704 & down & exonic & PAPPA \\
hsa_circ_0006697 & 0.00437 & 0.10841 & 2.41599 & down & exonic & RNF13 \\
hsa_circ_0001184 & 0.00051 & 0.04833 & 2.22426 & down & intronic & IFNGR2 \\
hsa_circ_0003645 & 0.00772 & 0.12396 & 2.10448 & down & exonic & C16orf62 \\
hsa_circ_0000997 & 0.00212 & 0.08204 & 2.02252 & down & exonic & SRBD1 \\
ha_circ0002316 & 0.00906 & 0.13053 & 1.90382 & down & exonic & OMA1 \\
hsa_circ_0000182 & 0.05013 & 0.23760 & 1.80159 & down & exonic & FLVCR1 \\
hsa_circ_0004115 & 0.00050 & 0.04833 & 1.79060 & down & exonic & AMD1 \\
\hline
\end{tabular}


identify the most clinically applicable biomarker, we chose the 3 circRNAs that showed the highest fold changes from the upregulated circRNAs (hsa_circ_0001874, hsa_circ_0001971, and hsa_circ_0008068) and 3 circRNAs that showed the highest fold changes from the downregulated circRNAs (hsa_circ_0000140, hsa_circ_0002632, and hsa_circ_0008792) for further validation.

\section{Validation of circRNAs expression}

To validate the circRNA microarray results, qRT-PCR was performed to analyze the selected circRNAs. The results showed that hsa_circ_0001874, hsa_circ_0001971, and hsa circ_0008068 levels were increased, whereas hsa_circ_0000140, hsa_circ_0002632, and hsa_

Fig. 1. Analysis of differentially expressed salivary circRNAs in OSCC patients $(n=3)$ and healthy controls $(n=3)$. (A) Hierarchical clustering results of circRNA expression profiles among the OSCC and healthy control groups. Red indicates high relative expression and green indicates low relative expression. (B) CircRNAs in the scatter plot above the top green line and below the bottom green line indicate more than a 1.5-fold change of circRNAs between the two groups. (C) Volcano plot of circRNA expression profile. The vertical lines correspond to 1.5 -fold upregulation and downregulation, and the horizontal line represents a P-value of 0.05 . The red point in the plot represents the differentially expressed circRNAs with statistical significance. OSCC: oral squamous cell carcinoma; HC: healthy control.

Fig. 2. Confirmation of the differential expression of circRNAs by qRT-PCR. The expression levels of six circRNAs were validated by qRT-PCR in the saliva from 90 OSCC patients and 82 healthy controls. The Mann-Whitney $U$ test was used for hsa_circ_0001874, hsa_circ_0001971, and hsa_circ_0008068; Student's t-test was used for hsa_circ_0000140, hsa_circ_0002632, and hsa circ_0008792. qRT-PCR data were analyzed by
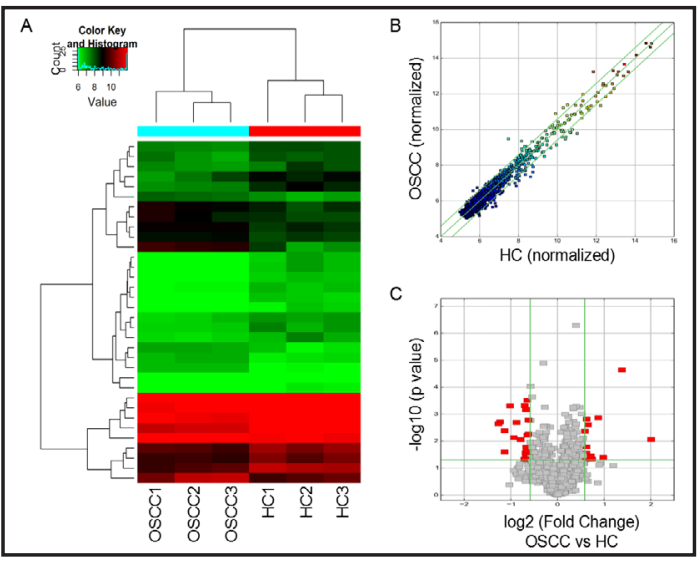
the $\Delta \mathrm{Ct}$ method, normalized against the internal control ( $\beta$-actin). Larger $\Delta$ Ct values indicate lower expression. Data are expressed as the mean \pm SEM. OSCC: oral squamous cell carcinoma; HC: healthy control.

Fig. 3. Sanger sequencing of hsa_circ_0001874

hsa_circ_0001971

hsa_circ_0008068

hsa_circ_0000140

hsa_circ_0002632

(E),

(A),

(B),

(C),

(D),

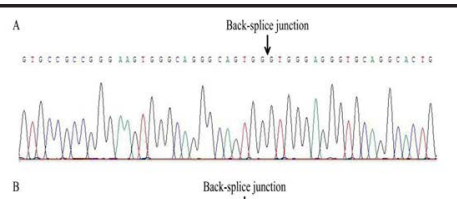
circ_0008792 (F) showed the backsplice junction.

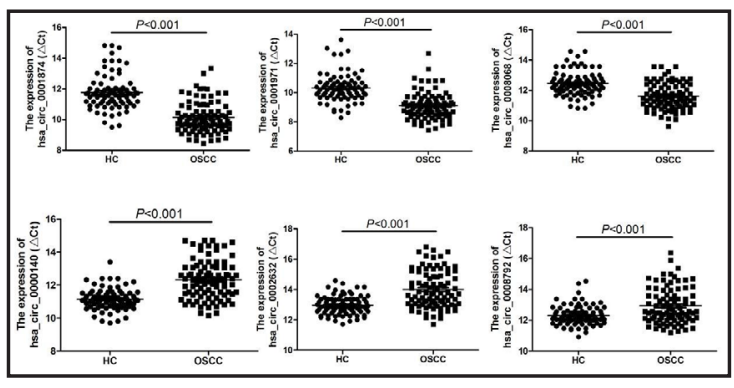


circ_0008792 levels were decreased in OSCC patients compared with the healthy controls $(P<0.001$; Fig. 2). These results were generally consistent with the microarray data. Sanger sequencing of the qRT-PCR products confirmed the back-splice junction sequence of these six circRNAs which were consistent with that from database (http://circinteractome.nia.nih. gov/) showed (Fig. 3).

\section{ROC curve analysis of differentially expressed circRNAs}

To compare the diagnostic value of these confirmed salivary circRNAs as candidate biomarkers of OSCC, ROC curve analysis was performed on data from the validation set. As shown in Fig. 4, the AUC was larger than 0.650 for all 6 candidate circRNAs, suggesting their potential diagnostic value. Notably, the AUC of hsa_circ_0001874 reached 0.863 (95\% confidence interval [CI]: 0.808-0.919, $P<0.001$ ), which was the largest of the 6 circRNAs. The AUC of the other circRNAs was 0.845 (95\% CI: 0.784-0.905, $P<0.001)$ for hsa_circ_0001971, 0.803 (95\% CI: 0.736-0.869, $P<0.0001$ ) for hsa_circ_0000140, 0.766 (95\% CI: 0.696-0.836, $P<0.001$ ) for hsa_circ_0002632, 0.757 (95\% CI: 0.685-0.830, $P<0.001$ ) for hsa_circ_0008068, and 0.658 (95\% CI: $0.577-0.740, P<0.001)$ for hsa_circ_0008792. The logistic regression model showed that the combination of hsa_circ_0001874 and hsa_circ_0001971 provided better diagnostic accuracy with an AUC of 0.922 (95\% CI: 0.883-0.961; $P<0.001$; Fig. 4G). The sensitivity and specificity of each circRNA were determined based on the cut-off value (Table 3).

Association between salivary circRNA expression and clinicopathological characteristics of OSCC patients

We also examined the association of these differentially expressed circRNAs with clinicopathological factors in all 90 OSCC patients. OSCC salivary samples were classified into high expression and low expression groups according to the median expression of the salivary circRNAs. As summarized in Table 4, the expression of salivary hsa_circ_0001874 was closely associated with TNM stage $(P=0.006)$ and tumor grade $(P=0.023)$, and the expression of salivary hsa_circ_0001971 was associated with TNM stage $(P=0.019)$. However, no significant correlations were evident between the expression levels of hsa_circ_0008068, hsa_circ_0000140, hsa circ_0002632, and hsa circ_00087921 and clinical factors (data not shown).

Table 3. Sensitivity and specificity of the candidate circRNAs in OSCC patients and healthy controls. Abbreviations: AUC: area under the curve

\begin{tabular}{ccccc}
\hline Candidate biomarkers & $\begin{array}{c}\text { AUC } \\
(95 \% \mathrm{CI})\end{array}$ & $\begin{array}{c}\text { Sensitivity }(\%) \\
(95 \% \mathrm{CI})\end{array}$ & $\begin{array}{c}\text { Specificity }(\%) \\
(95 \% \mathrm{Cl})\end{array}$ & P value \\
\hline hsa_circ_0001874 & $0.863(0.808-0.919)$ & $74.44(64.16-83.06)$ & $90.24(81.68-95.69)$ & $<0.0001$ \\
hsa_circ_0001971 & $0.845(0.784-0.905)$ & $75.56(65.36-84.00)$ & $87.80(78.71-93.99)$ & $<0.0001$ \\
hsa_circ_0008068 & $0.757(0.685-0.830)$ & $64.44(53.65-74.26)$ & $81.71(71.63-89.38)$ & $<0.0001$ \\
hsa_circ_0000140 & $0.803(0.736-0.869)$ & $71.11(60.60-80.18)$ & $81.71(71.63-89.38)$ & $<0.0001$ \\
hsa_circ_0002632 & $0.766(0.696-0.836)$ & $51.11(40.35-61.80)$ & $90.24(81.68-95.69)$ & $<0.0001$ \\
hsa_circ_0008792 & $0.658(0.577-0.740)$ & $43.33(32.92-54.20)$ & $85.37(75.83-92.20)$ & $=0.0003$ \\
hsa_circ_0001874+ & & & & \\
hsa_circ_0001971 & $0.922(0.883-0.961)$ & $92.68(84.75-97.27)$ & $77.78(67.79-85.87)$ & $<0.0001$ \\
\hline
\end{tabular}

Fig. 4. ROC curve analysis of hsa_circ_0001874 (A), hsa_circ_0001971 (B), hsa_circ_0008068 (C), hsa_circ_0000140 (D), hsa_circ_0002632 (E), hsa_ circ_0008792 (F), and hsa_circ_0001874 combined with hsa_circ_0001971 (G) in saliva from OSCC patients. AUC values are given on the graphs.

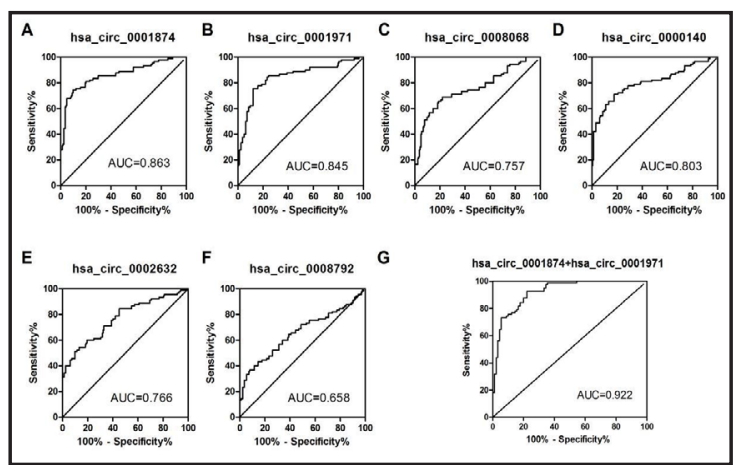


Table 4. Correlation between salivary hsa_circ_0001874 and hsa_circ_0001971 expression and clinicopathological characteristics of OSCC patients

\begin{tabular}{|c|c|c|c|c|c|c|c|c|}
\hline \multirow[b]{2}{*}{ Characteristics } & \multirow[b]{2}{*}{ Group } & \multirow[b]{2}{*}{ Total } & \multicolumn{2}{|c|}{ hsa_circ_0001874 expression } & \multirow[b]{2}{*}{$P$ value } & \multicolumn{2}{|c|}{ hsa_circ_0001971 expression } & \multirow[b]{2}{*}{$P$ value } \\
\hline & & & Low $(n=45)$ & High $(n=45)$ & & Low $(n=45)$ & High $(n=45)$ & \\
\hline \multirow[t]{2}{*}{ Gender } & Female & 37 & 17 & 20 & 0.520 & 19 & 18 & 0.830 \\
\hline & Male & 53 & 28 & 25 & & 26 & 27 & \\
\hline \multirow{2}{*}{ Age } & $\leq 60$ & 35 & 21 & 14 & 0.130 & 20 & 15 & 0.280 \\
\hline & $>60$ & 55 & 24 & 31 & & 25 & 30 & \\
\hline \multirow[t]{2}{*}{ Smoking status } & Non-smoker & 48 & 26 & 22 & 0.398 & 23 & 25 & 0.673 \\
\hline & Smoker & 42 & 19 & 23 & & 22 & 20 & \\
\hline \multirow[t]{2}{*}{ Tumor site } & Tongue & 32 & 15 & 17 & 0.660 & 18 & 14 & 0.378 \\
\hline & Non-tongue & 58 & 30 & 28 & & 27 & 31 & \\
\hline \multirow{2}{*}{ T stage } & T1-T2 & 57 & 32 & 25 & 0.126 & 31 & 26 & 0.274 \\
\hline & T3-T4 & 33 & 13 & 20 & & 14 & 19 & \\
\hline \multirow[t]{2}{*}{ Lymph node metastasis } & No & 55 & 30 & 25 & 0.280 & 32 & 23 & 0.052 \\
\hline & Yes & 35 & 15 & 20 & & 13 & 22 & \\
\hline \multirow[t]{2}{*}{ TNM stage } & I-II & 51 & 32 & 19 & 0.006 & 31 & 20 & 0.019 \\
\hline & III-IV & 39 & 13 & 26 & & 14 & 25 & \\
\hline \multirow[t]{2}{*}{ Tumor grade } & Well/moderate & 62 & 36 & 26 & 0.023 & 35 & 27 & 0.069 \\
\hline & Poor & 28 & 9 & 19 & & 10 & 18 & \\
\hline
\end{tabular}

Fig. 5. ROC curve analysis of the risk score of hsa_circ_0001874 and hsa_circ_0001971. (A) qRTPCR assay validation of hsa_circ_0001874 and hsa_ circ_0001971 expression levels in the saliva of 90 OSCC patients versus 70 OLK patients. The MannWhitney U test was used for statistical analysis. (B) ROC curve analysis of hsa_circ_0001874 and hsa_ circ_0001971 for the risk score in OSCC patients versus OLK patients. (C) ROC curve analysis of hsa circ_0001874 and hsa_circ_0001971 for the risk score in OSCC patients versus all controls (HCs and OLK). AUC values are given on the graphs. OSCC:

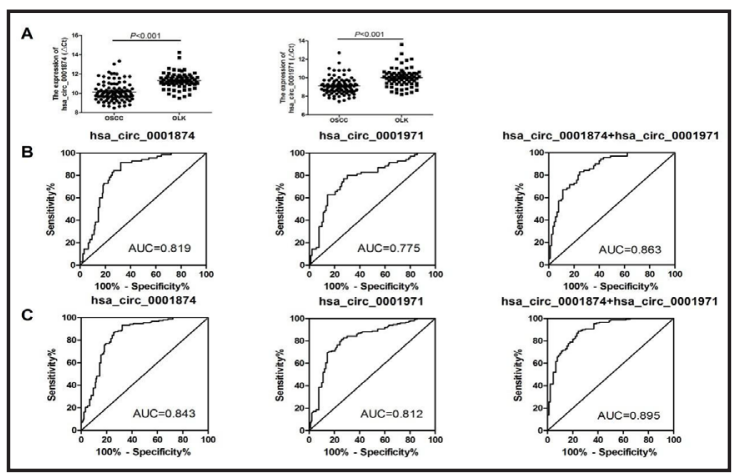
oral squamous cell carcinoma; HC: healthy control; OLK: oral leukoplakia.

Hsa_circ_0001874 and hsa_circ_0001971 expression in patients with OSCC and OLK

We analyzed the expression of salivary hsa_circ_0001874 and hsa_circ_0001971 in 90 OSCC patients and 70 OLK patients. The expression of hsa_circ_0001874 and hsa circ_0001971 was significantly increased in OSCC patients compared with OLK patients (all $P<0.001$; Fig. 5A). Subsequently, risk scores based on hsa_circ_0001874 and hsa_circ_0001971 were further assessed in OSCC patients and disease controls (OLK; Fig. 5B): the AUC for risk score based on hsa_circ_0001874 was 0.819 (95\% CI: 0.752-0.885; $P<0.001$ ), the AUC for risk score based on hsa_circ_0001971 was 0.775 (95\% CI: $0.700-0.849 ; P<0.001$ ), and the AUC for risk score based on both hsa_circ_0001874 and hsa_circ_0001971 was 0.863 (95\% CI: 0.808-0.918; $P<0.001)$. The risk score also significantly discriminated between patients with OSCC and all controls (healthy controls and OLK; Fig. 5C). The AUC for risk score based on hsa_circ_0001874 was 0.843 (95\% CI: 0.786-0.899; $P<0.001$ ), the AUC for risk score based on hsa_circ_0001971 was 0.812 (95\% CI: 0.754-0.871; $P<0.001)$, and the AUC for risk score based on both hsa_circ_0001874 and hsa_circ_0001971 was 0.895 (95\% CI: 0.8540.937; $P<0.001)$.

Correlation of salivary hsa_circ_0001874 and hsa_circ_0001971 expression levels between preoperative and postoperative patients with OSCC

The postoperative saliva samples from 25 patients were collected 1 month after surgery. The expression levels of salivary hsa_circ_0001874 and hsa_circ_0001971 were significantly decreased in postoperative samples compared with preoperative samples $(P<0.001)$ (Fig. 6). Compared with healthy controls, the mean levels of hsa_circ_0001874 and hsa_circ_0001971 returned to nearly normal after surgical treatment, but with no significant difference between postoperative and healthy controls $(P>0.05)$. 
Fig. 6. Comparison of salivary hsa_circ_0001874 and hsa_circ_0001971 concentrations between preoperative and postoperative samples from OSCC patients. Changes of (A) hsa_circ_0001874 and (B) hsa_circ_0001971 salivary levels in patients with OSCC ( $\mathrm{n}=25)$ prior to (preoperative) and 1 month following (1 month postoperative) surgical removal of the tumor are shown. The Wilcoxon test was used to compare the paired saliva samples before and 1 month after surgery. The value between the $\mathrm{HC}(\mathrm{n}=82)$ and postoperative group $(\mathrm{n}=25)$ was not significantly different. qRT-PCR data were analyzed

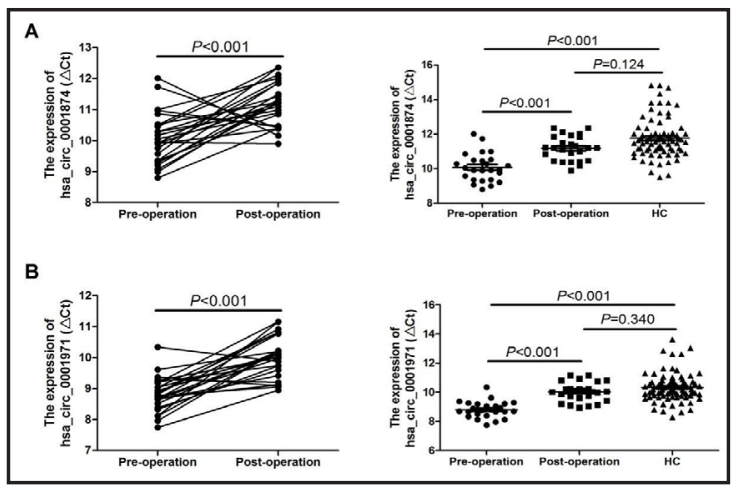
by the $\Delta \mathrm{Ct}$ method and normalized against the internal control ( $\beta$-actin). Larger $\Delta$ Ct values indicate lower expression. OSCC: oral squamous cell carcinoma; HC: healthy control.

\section{Discussion}

Even though great progress has been made in the early diagnosis, surgical technique, radiation, and chemotherapy of OSCC, the prognosis of patients with this disease is still not ideal due to the lack of non-invasive, rapid, and accurate signatures for OSCC early screening [20]. Salivary screening is currently the best choice as the primary screening test for highrisk cases of OSCC because the collection procedure is noninvasive and low cost [21,22]. Several studies have demonstrated the feasibility of using salivay miRNAs and long noncoding RNAs (lncRNAs) as potential biomarkers of OSCC $[23,24]$. CircRNAs are a naturally occurring type of universal and diverse endogenous non-coding RNA. They are abundant, conserved across species, stably expressed in saliva, blood, and exosomes, and exhibit tissueand developmental stage-specific expression, all of which demonstrate their promise as cancer biomarkers. In addition, circRNAs form a covalently closed continuous loop without $5^{\prime}$ caps and 3' poly(A) tails, which make them resistant to RNase R activity and more stable than linear lncRNAs. The average half-life of circRNAs in most species is more than $48 \mathrm{~h}$, whereas, on average, the half-life of mRNAs is about $10 \mathrm{~h}$ [25]. Studies have shown great potential of circulating circRNAs as powerful and non-invasive biomarkers in a number of diseases [26, 27]. However, the diagnostic performance of saliavry circRNAs in OSCC has not been explored previously.

In this study, we performed a microarray analysis of dysregulated circRNAs by comparing the transcriptome profiles of saliva from patients with OSCC and healthy controls. Compared with healthy controls, we found 12 upregulated and 20 downregulated circRNAs in the saliva of OSCC patients. To identify clinically applicable biomarkers, we detected 6 significantly differentially expressed salivary circRNAs and investigated the association between their expression levels and clinicopathological characteristics of OSCC patients. The validation study demonstrated that salivary levels of hsa_circ_0001874, hsa_circ_0001971, and hsa_ circ_0008068 were remarkably increased, and those of hsa_circ_0000140, hsa_circ_0002632, and hsa_circ_0008792 were remarkably decreased in these patients.

ROC curves were constructed and used to evaluate the discriminatory power of salivary circRNAs for differentiating between OSCC and healthy controls. ROC curve analysis suggested that hsa_circ_0001874 has significant value for OSCC diagnosis, followed by hsa_circ_0001971, hsa_circ_0000140, hsa_circ_0002632, hsa_circ_0008068, and hsa circ_0008792. The logistic regression model demonstrated that the combination of hsa circ_0001874 and hsa_circ_0001971 have a higher AUC than the individual circRNAs. In addition, we also found that the high expression level of hsa_circ_0001874 was associated with TNM stage and tumor grade, and that the expression level of hsa_circ_0001971 was associated with TNM stage. Risk score based on hsa_circ_0001874 and hsa_circ_0001971 
also demonstrated that they could significantly discriminate patients with OSCC from those with OLK. These results indicate that the altered expression of salivary hsa_circ_0001874 and hsa_circ_0001971 may be related to their involvement in the development and progression of OSCC.

Studies have revealed that circRNAs can serve as a miRNA sponge and can interact with miRNA, resulting in an imbalance between miRNA and its target genes, which leads to the initiation and progression of a variety of diseases including cancer [28, 29]. The relationship between miRNAs and OSCC suggest that circRNAs might be involved in the pathogenesis and progression of OSCC. Recently, Chen et al [30]. reported that circRNA_100290 absorbed to miR-29, thereby relieving the inhibition of cell division protein kinase 6 and promoting the proliferation of OSCC. To evaluate the potential functions of hsa_circ_0001874 and hsa circ_0001971, the circRNA/miRNA interaction was predicted using Arraystar's homemade miRNA target prediction software based on TargetScan and miRanda. We found that the potential miRNAs targets of hsa_circ_0001874 included miR-661, miR-662, miR-593-5p, miR-107, and miR-103a-3p, and those of hsa_circ_0001971 included miR-152-5p, miR103a-3p, miR-107, miR-505-3p, and miR-9-5p. It is worth noting that miR-107 and miR103a-3p play vital roles in cell division, metabolism, stress response, and angiogenesis [31]. In addition, the dysregulation of miR-107 and miR-103a-3p are involved in the development and progression of multiple tumors [32,33]. However, due to the limited knowledge of the functions of circRNAs and miRNAs, many of the circRNA/miRNA interactions should be analyzed in future studies.

Several limitations in this study should be acknowledged. First, circRNAs in tissue and plasma were not detected and therefore the circRNA profiles of tissue, plasma, and saliva from the same patient could not be compared simultaneously. Second, the sample size was relatively small and the subjects were limited to a Chinese Han population. Thus, it is necessary to expand the sample size and conduct a multi-center study to validate our findings. Finally, we did not investigate the exact role of circRNAs in OSCC tumorigenesis.

\section{Conclusion}

We identified differentially expressed circRNAs in saliva from subjects with OSCC and healthy controls. Furthermore, hsa_circ_0001874 and hsa_circ_0001971 were identified as potential molecular markers for the diagnosis of OSCC. To the best of our knowledge, this is the first study to address salivary circRNA expression profiles in OSCC patients. Further studies are needed on the correlation of the biological function of circRNAs in OSCC, which may be involved in OSCC tumorigenesis and serve as potential novel targets for the early diagnosis, prognostic evaluation, and therapy of patients with OSCC.

\section{Acknowledgements}

We thank all donors who participated in work. This work was supported by grants from the National Natural Science Foundation of China (Grant No. 81660444), the Foundation for Distinguished Young Scientists of Jiangxi Province (Grant No. 20171BCB23087), the Province Natural Science Foundation of Jiangxi Province (Grant No. 20171BAB205050), and Jiangxi Key Research and Development Program (Grant No. 20161BBG70149).

\section{Disclosure Statement}

The authors have no conflict of interests to declare. 


\section{Cellular Physiology Cell Physiol Biochem 2018;47:2511-2521 \begin{tabular}{l|l} 
and Biochemistry Published online July 10, 2018 & $\begin{array}{l}\text { C) } 2018 \text { The Author(s). Published by S. Karger AG, Basel } \\
\text { www.karger.com/cpb }\end{array}$ \\
\hline
\end{tabular}}

Zhao et al.: Salivary CircRNAs and OSCC

\section{References}

1 Sasahira T, Kirita T, Kuniyasu H: Update of molecular pathobiology in oral cancer: a review. Int J Clin Oncol 2014;19:431-436.

- Brocklehurst PR, Baker SR, Speight PM: Oral cancer screening: what have we learnt and what is there still to achieve? Future Oncol 2010;6:299-304.

- 3 Irimie AI, Braicu C, Sonea L, Zimta AA, Cojocneanu-Petric R, Tonchev K, Mehterov N, Diudea D, Buduru S, Berindan-Neagoe I: A Looking-Glass of Non-coding RNAs in oral cancer. Int J Mol Sci 2017;18:

4 Qu S, Yang X, Li X, Wang J, Gao Y, Shang R, Sun W, Dou K, Li H: Circular RNA: A new star of noncoding RNAs. Cancer Lett 2015;365:141-148.

-5 Qu S, Zhong Y, Shang R, Zhang X, Song W, Kjems J, Li H: The emerging landscape of circular RNA in life processes. RNA Biol 2017;14:992-999.

-6 Chen LL: The biogenesis and emerging roles of circular RNAs. Nat Rev Mol Cell Biol 2016;17:205-211.

7 Zhao J, Li L, Wang Q, Han H, Zhan Q Xu M: CircRNA Expression Profile in Early-Stage Lung Adenocarcinoma Patients. Cell Physiol Biochem 2017;44:2138-2146.

8 Bezzi M, Guarnerio J, Pandolfi PP: A circular twist on microRNA regulation. Cell Res 2017;27:1401-1402.

-9 Rong D, Sun H, Li Z, Liu S, Dong C, Fu K, Tang W, Cao H: An emerging function of circRNA-miRNAs-mRNA axis in human diseases. Oncotarget 2017;8:73271-73281.

10 He J, Xie Q Xu H, Li J, Li Y: Circular RNAs and cancer. Cancer Lett 2017;396:138-144.

11 Fan X, Weng X, Zhao Y, Chen W, Gan T, Xu D: Circular RNAs in Cardiovascular Disease: An Overview. Biomed Res Int 2017;2017:5135781.

12 Li TR, Jia YJ, Wang Q, Shao XQ, Lv RJ: Circular RNA: a new star in neurological diseases. Int J Neurosci 2017;127:726-734.

13 Chen Y, Li C, Tan C, Liu X: Circular RNAs: a new frontier in the study of human diseases. J Med Genet 2016;53:359-365.

14 Zhang Y, Liang W, Zhang P, Chen J, Qian H, Zhang X, Xu W: Circular RNAs: emerging cancer biomarkers and targets. J Exp Clin Cancer Res 2017;36:152.

15 Zhao Z, Li X, Gao C, Jian D, Hao P, Rao L, Li M: Peripheral blood circular RNA hsa_circ_0124644 can be used as a diagnostic biomarker of coronary artery disease. Sci Rep 2017;7:39918.

16 Yin WB, Yan MG, Fang X, Guo JJ, Xiong W, Zhang RP: Circulating circular RNA hsa_circ_0001785 acts as a diagnostic biomarker for breast cancer detection. Clin Chim Acta 2017;10.1016/j.cca.2017.10.011

17 Saxena S, Sankhla B, Sundaragiri KS, Bhargava A: A Review of Salivary Biomarker: A Tool for Early Oral Cancer Diagnosis. Adv Biomed Res 2017;6:90.

18 Bahn JH, Zhang Q, Li F, Chan TM, Lin X, Kim Y, Wong DT, Xiao X: The landscape of microRNA, Piwi-interacting RNA, and circular RNA in human saliva. Clin Chem 2015;61:221-230.

19 Wang MC, Li S: Bivariate marker measurements and ROC analysis. Biometrics 2012;68:1207-1218.

20 Blatt S, Kruger M, Ziebart T, Sagheb K, Schiegnitz E, Goetze E, Al-Nawas B, Pabst AM: Biomarkers in diagnosis and therapy of oral squamous cell carcinoma: A review of the literature. J Craniomaxillofac Surg 2017;45:722730.

-21 Sannam Khan R, Khurshid Z, Akhbar S, Faraz Moin S: Advances of Salivary Proteomics in Oral Squamous Cell Carcinoma (OSCC) Detection: An Update. Proteomes 2016;4:

-22 Wu JY, Yi C, Chung HR, Wang DJ, Chang WC, Lee SY, Lin CT, Yang YC, Yang WC: Potential biomarkers in saliva for oral squamous cell carcinoma. Oral Oncol 2010;46:226-231.

-23 Dumache R: Early Diagnosis of Oral Squamous Cell Carcinoma by Salivary microRNAs. Clin Lab 2017;63:17711776.

24 Tang H, Wu Z, Zhang J, Su B: Salivary lncRNA as a potential marker for oral squamous cell carcinoma diagnosis. Mol Med Rep 2013;7:761-766.

25 Jeck WR, Sharpless NE: Detecting and characterizing circular RNAs. Nat Biotechnol 2014;32:453-461.

26 Rong D, Tang W, Li Z, Zhou J, Shi J, Wang H, Cao H: Novel insights into circular RNAs in clinical application of carcinomas. Onco Targets Ther 2017;10:2183-2188.

27 Kulcheski FR, Christoff AP, Margis R: Circular RNAs are miRNA sponges and can be used as a new class of biomarker. J Biotechnol 2016;238:42-51.

-28 Militello G, Weirick T, John D, Doring C, Dimmeler S, Uchida S: Screening and validation of lncRNAs and circRNAs as miRNA sponges. Brief Bioinform 2017;18:780-788. 
Cellular Physiology Cell Physiol Biochem 2018;47:2511-2521 \begin{tabular}{l|l} 
and Biochemistry Published 10.1159/000491624 & $\begin{array}{l}\text { D) } 2018 \text { The Author(s). Published by S. Karger AG, Basel } \\
\text { www.karger.com/cpb }\end{array}$ \\
\hline
\end{tabular}

Zhao et al.: Salivary CircRNAs and OSCC

29 Cortes-Lopez M, Miura P: Emerging Functions of Circular RNAs. Yale J Biol Med 2016;89:527-537.

30 Chen L, Zhang S, Wu J, Cui J, Zhong L, Zeng L, Ge S: circRNA_100290 plays a role in oral cancer by functioning as a sponge of the miR-29 family. Oncogene 2017;36:4551-4561.

-31 Jiang ZP, Zhou TB: Role of miR-107 and its signaling pathways in diseases. J Recept Signal Transduct Res 2014;34:338-341.

-32 Sharma P, Saini N, Sharma R: miR-107 functions as a tumor suppressor in human esophageal squamous cell carcinoma and targets Cdc42. Oncol Rep 2017;37:3116-3127.

-33 Liang J, Liu X, Xue H, Qiu B, Wei B, Sun K: MicroRNA-103a inhibits gastric cancer cell proliferation, migration and invasion by targeting c-Myb. Cell Prolif 2015;48:78-85. 